\title{
Experimental Studies of High-Accuracy RFID Localization with Channel Impairments
}

\author{
Eric Pauls and Yimin D. Zhang \\ RFID Laboratory, Center for Advanced Communications, Villanova, PA 19085, USA
}

\begin{abstract}
Radio frequency identification (RFID) systems present an incredibly cost-effective and easy-to-implement solution to close-range localization. One of the important applications of a passive RFID system is to determine the reader position through multilateration based on the estimated distances between the reader and multiple distributed reference tags obtained from, e.g., the received signal strength indicator (RSSI) readings. In practice, the achievable accuracy of passive RFID reader localization suffers from many factors, such as the distorted RSSI reading due to channel impairments in terms of the susceptibility to reader antenna patterns and multipath propagation. Previous studies have shown that the accuracy of passive RFID localization can be significantly improved by properly modeling and compensating for such channel impairments. The objective of this paper is to report experimental study results that validate the effectiveness of such approaches for high-accuracy RFID localization. We also examine a number of practical issues arising in the underlying problem that limit the accuracy of reader-tag distance measurements and, therefore, the estimated reader localization. These issues include the variations in tag radiation characteristics for similar tags, effects of tag orientations, and reader RSS quantization and measurement errors. As such, this paper reveals valuable insights of the issues and solutions toward achieving high-accuracy passive RFID localization.
\end{abstract}

Keywords: RFID localization, multilateration, received signal strength indicator, channel impairment

\section{INTRODUCTION}

The field of radio frequency identification (RFID) has seen incredible growth in recent years due to low hardware costs, well-defined standards for universal compatibility, and accessible pricing. The rapid growth of RFID technology is fueled by its adoption by major retail and industrial companies and the U.S. Department of Defense [1]. RFID technology, which allows wireless unique identification of an object, has evolved from simple asset identification to precise tracking and localization for mobile robots [2].

A typical RFID system consists of RFID readers, reader antennas, and RFID tags. The reader "polls" for present tags by radiating electromagnetic waves through the reader antenna. Tags, which consist of a compact integrated circuit and antenna supported by an application-specific body, receive some of the transmitted waves and send back their unique identifying code by means of backscattering [3,4]. There are several variations of tag types to fit specific applications and transmission regulations. In this paper, we employ passive ultra-high frequency (UHF) tags. These tags are popular for localization solutions as they require no power supply other than radiation from the reader, and can operate reliably for a range of up to a few meters.

Localization through RFID is achieved by using an RFID system to sense a certain reader-tag arrangement and then deducing position information from this measurement. In this paper, we discuss a popular method of RFID localization based on the signal strength of the backscattered radiation and explore the principle factors that affect localization accuracy, discovered through an experimental study. We then present a simple, highly accurate localization method based on the findings. We introduce the concept of received signal strength indicator (RSSI) metric and its important role in RFID localization systems. We then present a series of experiments to understand the reliability of the RSSI measurement data and the behavior of RSSI as it relates to distance and tag orientation and derive a model for this relationship. From this experimental study, we introduce an RSSI-based localization scheme and analyze its performance with an experimental evaluation. We conclude with a summary of our findings and suggestions for further improvement of the localization system. 


\section{RSSI-BASED LOCALIZATION}

RFID localization algorithms are designed to determine the position of an RFID reader or RFID tag in a region of interest. There are five major categories of RFID localization methods that can be used to exploit the available information in a tag-reader system: trilateration/multilateration, triangulation, hybrid direction/range methods, radio map matching methods, and proximity sensing [5]. Trilateration/multilateration relies on range information from three or more distinct reference points. Triangulation relies on the direction-of-arrival (DOA) of signals from two or more reference points [6]. The hybrid direction/range method exploits both an estimated DOA and estimated range information from a single or multiple known points. Radio map matching is a method in which a "scene" of reference tags is surveyed and stored as a radio map. When a new tag is introduced into the scene, the measurement data for the new tag is compared to the radio map to estimate its position [7]. Proximity based algorithms couple the prior knowledge about the read range between a reader and the position of a reference tag/reader to predict a region within which the reader/tag may be located. When multiple reference tags are available, the area of intersection of the multiple "predicted" regions indicates the possible location of the reader. As such, the accuracy of localization can be improved through a dense deployment of the reference tags.

Range information is commonly obtained through the received signal strength indicator reported from the reader with each successful read of a tag. RSSI is a measure of the received power of a radio transmission. In the case of passive UHF RFID, RSSI is related to the ratio of the received power from a responding tag to the transmitted power from the RFID reader. RSSI-based localization techniques for RFID have become a popular choice, as the RSSI measurement offers significantly more information about the location of a tag than simply recording whether a tag is visible or not visible. The RSSI-based localization techniques vary from purely theoretical, physics-based approaches [8] to empirical models based on experiments and machine learning [9].

Signal power in a two-way line-of-sight RFID transmission as a function of distance can be modeled using the Friis transmission equation, expressed as

$$
P_{\mathrm{Rx}}=P_{\mathrm{Tx}} G_{\mathrm{tag}}^{2} G_{\mathrm{read}}^{2}\left(\frac{\lambda}{4 \pi d}\right)^{4},
$$

where $P_{\mathrm{Rx}}$ and $P_{\mathrm{Tx}}$ are the received and transmitted power at the reader antenna, $G_{\text {tag }}$ and $G_{\text {read }}$ are the gains of the tag and reader antennas, $d$ is the distance between the reader antenna and the tag, and $\lambda$ is the wavelength of the signal. To improve the accuracy of the transmission equation, the gain of the reader antenna and the tag antenna can be expressed as functions of direction angles, and the power efficiency of the tag can be expressed as a constant $\eta$, which is typically about $1 / 3[4$, p. 77$]$. That is,

$$
P_{\mathrm{Rx}}=P_{\mathrm{Tx}} \eta G_{\mathrm{tag}}^{2}\left(\theta_{\mathrm{tag}}\right) G_{\mathrm{read}}^{2}\left(\theta_{\text {read }}\right)\left(\frac{\lambda}{4 \pi d}\right)^{4} .
$$

In the effective reading range of an RFID reader/antenna system operating at the limit transmission power allowed by the United States Federal Communications Commission (FCC), which is 4 Watts or $36 \mathrm{dBm}$, the transmit/receive power ratio is expected to be between $-10 \mathrm{dBm}$ and $-100 \mathrm{dBm}$ [4]. As there is no standard for RSSI measurements, manufacturers of RFID readers can choose to report RSSI on an arbitrary scale. The actual RSSI value reported from a reader may be heavily manipulated from the original power ratio. For example, in the Alien ALR-9680 RFID reader model used in this study, the RSSI is mapped to a linear scale with respect to the range. In practical applications of RSSI-based localization, it is important to understand the relationship between the reader's RSSI value and the true power measurement $P_{\mathrm{Rx}}$.

With an accurate model for RSSI as a function of position, it is possible to combine RSSI measurements from multiple tags to localize an RFID reader. In this paper, we explore the process of generating an RSSI model, which is then applied to a localization problem to precisely estimate the position of a reader in a field of tags.

\section{TAG READING EXPERIMENTS}

To understand and characterize the performance of RFID systems and to achieve accurate RSSI measurements, we conducted experiments using an RFID reader and RFID tags to examine the RSSI metric in terms of the tag performance, manufacturing differences between tags, and changes in RSSI with different positions and orientations of the tags. Experimental studies on the variation in performance across different tags, the measurement error associated 
with each RSSI recorded, and the effects of tag mounting surfaces on RSSI reveal several potential sources of error in RSSI-based RFID localization. In this section, we present experimental results of RSSI measurements, and discuss possible sources of RSSI errors and remedy solutions.

\subsection{Experimental Setting}

The reader setup for the experiments includes an Alien ALR-9680 RFID reader with transmit power of $1 \mathrm{~W}$ (30 dBm) communicating in the UHF frequency band between 902.75-927.25 MHz. The reader is connected to a Poynting PatchA0026 right hand circularly polarized antenna with a nominal gain of $6.5 \mathrm{dBi}( \pm 0.5 \mathrm{~dB})$ and $3 \mathrm{~dB}$ beamwidth of $60^{\circ}\left( \pm 5^{\circ}\right)$ and $74^{\circ}\left( \pm 5^{\circ}\right)$ in elevation and azimuth, respectively. Alien Squiggle Higgs3 passive UHF RFID tags are used for all experiments. As shown in Fig. 1, the experiments were performed on a wooden frame designed to hold the antenna and tags in specific locations in space while minimizing electromagnetic reflections. Additionally, electromagnetic absorbing mats were placed on the floor beneath the test setup to eliminate reflections from the laboratory floor.

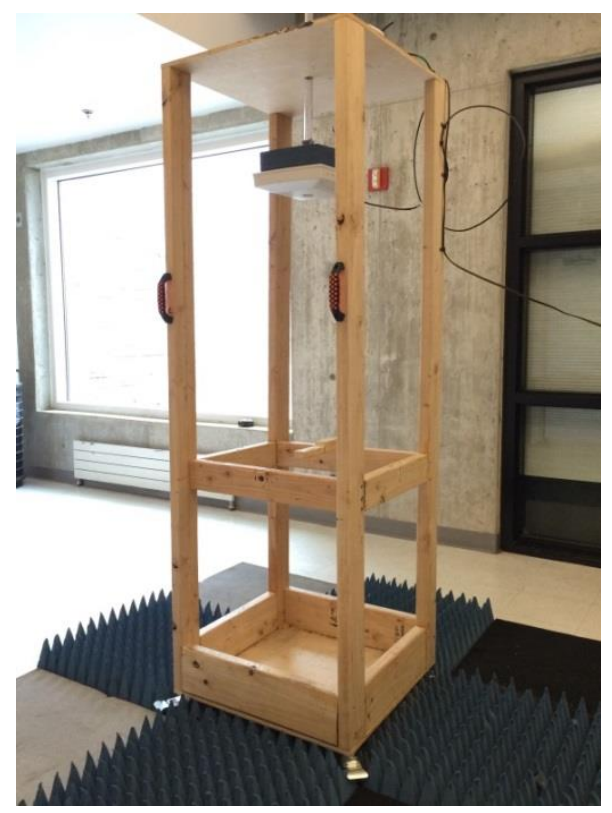

Figure 1. RFID test environment: Wooden frame holding reader, antenna, and an RFID tag above a floor of electromagnetic absorbers.

\subsection{Tag RSSI Performance}

We first perform an experiment on tags at a fixed position to observe the variation in RSSI measurements over 500 repeated reads. The measurement data for a single tag at a distance of $76 \mathrm{~cm}$ (20 inches) is shown in Fig. 2 for the first 100 reads. It is clear that the RSSI measurement varies significantly over the different reads. It should also be noted that the RSSI measurements are quantized by the reader to integer values with no measurement unit.

The experiment was then repeated with 45 tags at 500 reads each to study the differences in RSSI performance between different tags, and the results are shown in Fig. 3. It is observed from Fig. 3(a) that all of the 45 tags tested show very similar standard deviation of about 1.57. The mean RSSI for each tag, however, varies significantly in this experiment. Fig. 3(b) shows the histogram of the mean RSSI for the 45 tags, which clearly demonstrate that the same type of tags from the same manufacturer can still perform differently. Therefore, to effectively account for both the variation in RSSI measurements for a specific tag and the variation in performance between different tags, the tags should be "prescreened" to select only tags that perform similarly when tested in the same setting. This process is described in [10] as a method of increasing accuracy of RSSI measurements. For this experiment, we chose tags within the 92-92.25 RSSI range at a distance of 30 inches. With a set of similarly performing tags selected, the number of tag reads to average together can be chosen to achieve a desired accuracy of the mean RSSI. Fig. 4 shows an example of the cumulative progression of mean RSSI for 4 tags as the number of tag reads increases. The RSSI for each tag converges to within 
\pm 0.25 of the final mean in about 37 samples.

To examine the number of reads necessary to arrive at a reasonable approximation of the mean RSSI, Fig. 4 shows the progression of the cumulative mean of the RSSI measurements for each tag. It is observed that the averaged RSSI converges approximately after 15 reads with a standard deviation of 1.65 . Recording RSSI as the mean value of several measurements helps to eliminate both measurement noise and the quantization noise caused by the reader's integer rounding.

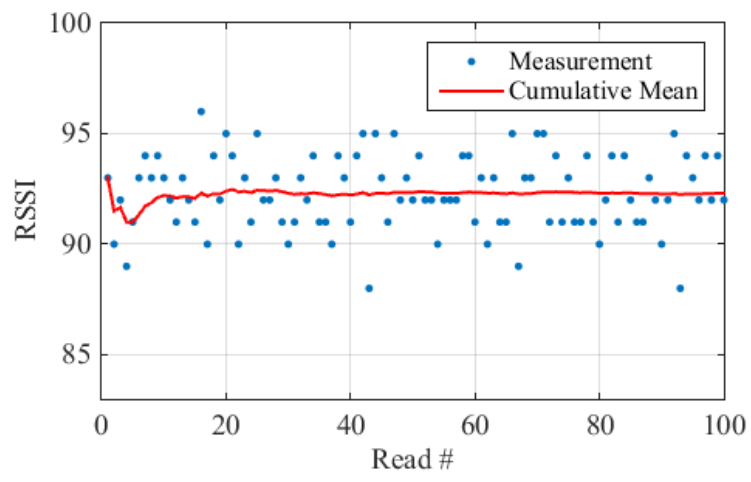

Figure 2. Variations in RSSI readings for a single fixed tag.

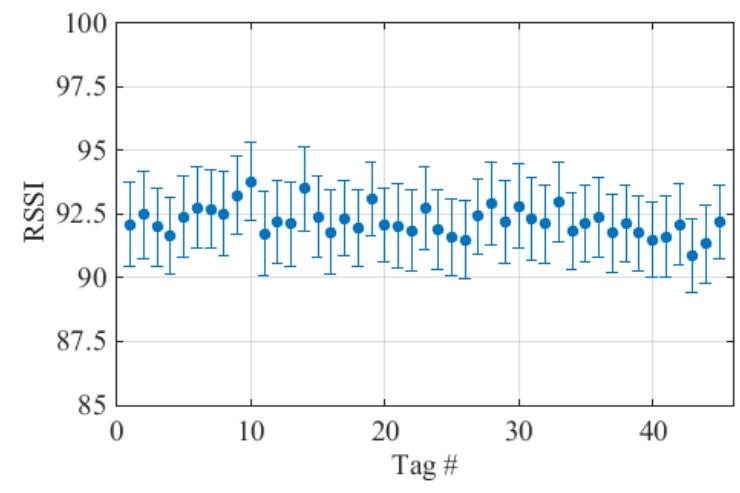

(a)

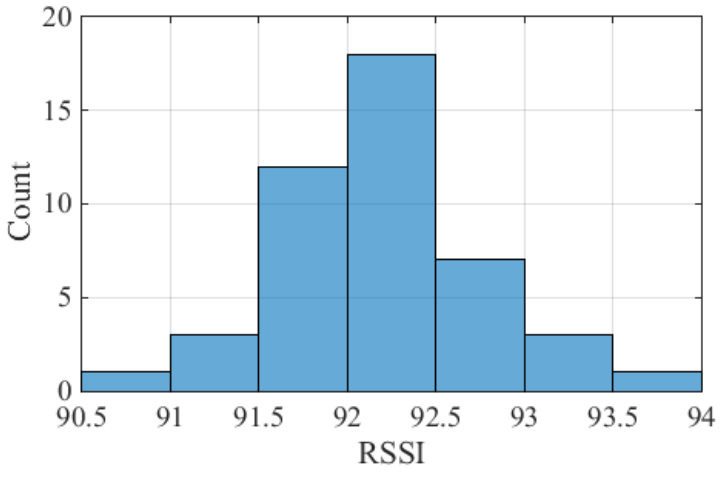

(b)

Figure 3. RSSI measurements over 45 tags (500 measurements per tag). (a) error-bar plot with mean and standard deviation; (b) histogram.

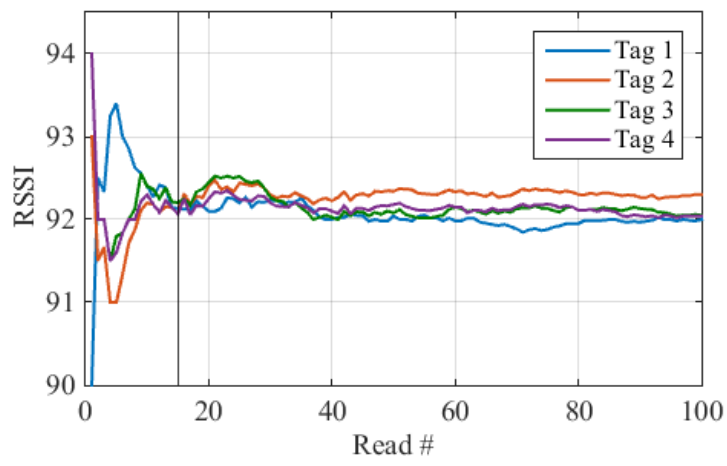

Figure 4. Progression of cumulative mean RSSI for 4 RFID tags. 


\subsection{RSSI versus Distance between RFID Reader and Tag}

To study the relationship between the RSSI and the distance between the RFID reader and tag, we place an RFID tag underneath the RFID reader antenna with a varied distance. Fig. 5 shows the RSSI mean and standard deviation over 500 reads with the reader-tag distance varying from $30 \mathrm{~cm}$ (12 inches) to $1.94 \mathrm{~m}$ (76.5 inches). A similar experiment was performed in [10] to create an empirical spatial model of RSSI.

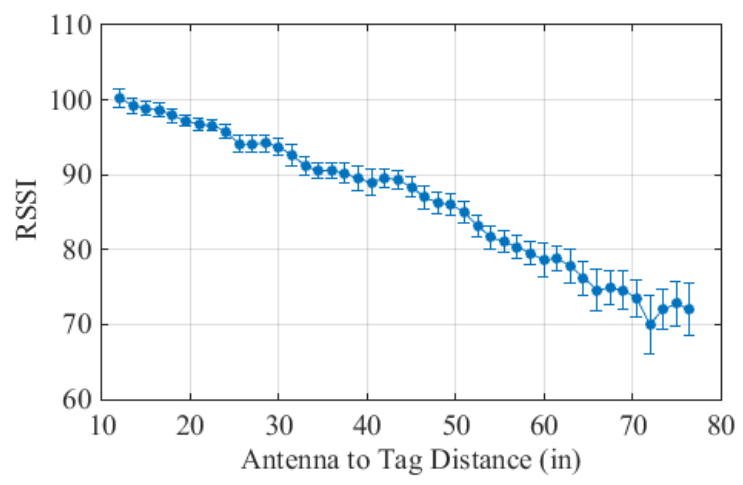

Figure 5. RSSI versus distance between reader antenna and tag.

This experiment shows that the reader's reported RSSI varies linearly with distance. Additionally, the standard deviation for RSSI measurements increases with distance. Small variations in the data can be attributed to small amounts of multipath interference from the test environment. It should be noted that this linear relationship is caused by a linearization algorithm (discussed in Section 3.4) that is specific to the RFID reader.

\subsection{RSSI versus Tag Orientation Angle}

The Alien Squiggle tags, as well as many other commonly used RFID tags, are of a dipole design. Therefore, their transmission/receive strength can be expected to vary significantly with changes in angle. The gain decreases as the axis of the tag becomes more closely aligned with the line of sight to the reader because of the toroid-shaped radiation pattern of the tag. The angle $\theta$ (shown in Fig. 6(a)) is defined as the orientation angle of the tag relative to the reader-tag line of sight. For a given reader and tag setup, $\theta$ is calculated as

$$
\theta=\cos ^{-1}\left(\frac{\vec{a} \cdot \vec{b}}{\|\vec{a}\|\|\vec{b}\|}\right),
$$

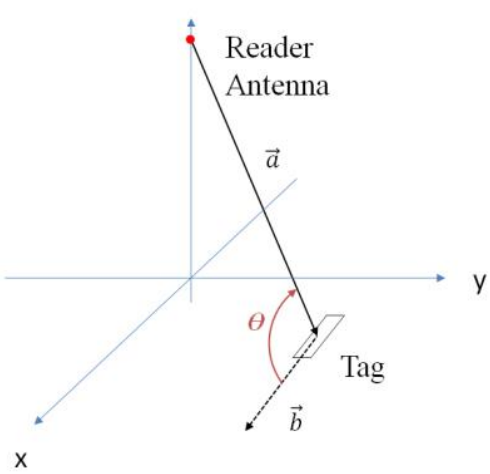

(a)

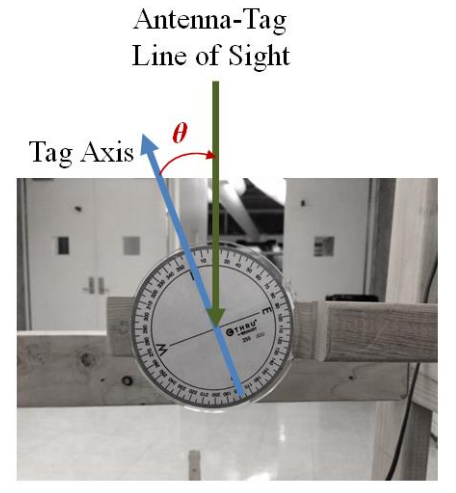

(b)

Figure 6. (a) Depiction of $\theta$, the three-dimensional angle between the tag antenna axis and the line of sight from the reader antenna to the tag. (b) Experiment setup for measuring RSSI while varying $\theta$. 
where $\vec{a}$ is the line of sight unit vector from the reader to the tag, and $\vec{b}$ is a unit vector in the direction of the tag's axis. To study the relationship between the RSSI and the orientation angle $\theta$, we place an RFID tag beneath the RFID reader antenna at a fixed distance of $76 \mathrm{~cm}$ (30 inches) and rotate the tag such that $\theta$ increases in increments of $5^{\circ}$. The results of this experiment in Fig. 7 show that the orientation angle $\theta$ has a significant effect on the RSSI. Similar to an ideal dipole, the directive signal strength is sinusoidal and approximately symmetric about $0^{\circ}$. Accounting for the change in RSSI with respect to $\theta$ allows for significant improvement over a traditional multilateration approach.

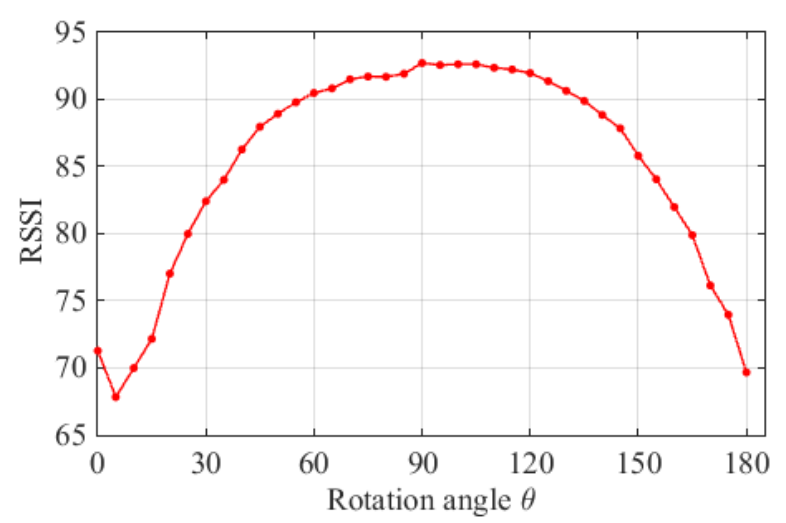

Figure 7. Average of 500 RSSI reads versus tag angle $\theta .0^{\circ}$ indicates that the tag axis is perpendicular to the line of sight to the reader antenna.

\subsection{RSSI Transmission Model}

The data from Sections 3.2 and 3.3 allow us to create a model for RSSI as a function of orientation angle and distance in the form of Equation (2). We first account for the linearization performed by the RFID reader. We observe that RSSI reported from the reader varies linearly with distance, which is achieved by performing a transformation on the received signal power measurement

$$
R S S I=F-\left(\frac{P_{R x}}{P_{T x}}\right)^{-1 / 4}
$$

By substituting the equation for received power $P_{\mathrm{Rx}}$ from (2), we derive the equation for mapping the RFID reader's RSSI measurement to signal power to be

$$
\operatorname{RSSI}(d, \theta)=F-\left(\frac{A \times G_{t a g}(\theta)}{d^{4}+k}\right)^{-1 / 4}
$$

where $F$ is an additive constant within the reader, $k$ is a small constant to account for nonlinearities in the reader's measurements, and $A$ is a scaling factor that encompasses the term $\left(\frac{\lambda}{4 \pi}\right)^{4}, \operatorname{tag} \operatorname{loss} \eta$, and $G_{\text {read }}$, which is assumed to be constant within the main beam of the reader antenna. As the tag's directive gain is very close to that of an ideal dipole, we can model $G_{\text {tag }}(\theta)$ as

$$
G_{\mathrm{tag}}(\theta) \propto \mid\left(\left.\sin (\theta-\varphi)\right|^{b},\right.
$$

with phase adjustment $\varphi$ and exponent $b$ to account for the small differences between an ideal dipole and the Alien Squiggle inlay antenna. Equations (5) and (6) yield a complete characterization of RSSI with respect to distance and tag direction. By fitting equation (5) to the experimental data for distance and angle, we find the values for the RSSI equation constants as

$$
\operatorname{RSSI}(d, \theta)=F-\left(\frac{A \times \mid\left(\left.\sin (\theta-\varphi)\right|^{b}\right.}{d^{4}+k}\right)^{-1 / 4},
$$

where the constants are summarized in the following table: 


\begin{tabular}{|c|c|c|c|c|}
\hline$F$ & $A$ & $k$ & $\varphi$ & $b$ \\
\hline 109 & $1.363 \times 10^{5}$ & $3.445 \times 10^{-7}$ & $5^{\circ}$ & 2.5 \\
\hline
\end{tabular}

\subsection{Remarks}

The RSSI measurement data from these tags reveal the following important facts:

1. Individual RSSI measurement of a tag varies from sample to sample within a small range. However, the measurements quickly converge to a consistent mean.

2. RSSI varies from tag to tag due to variations in manufacturing quality.

3. The variance of RSSI measurements is consistent across different tags. The RSSI measurements for a set of tags can be expected to converge to a desired margin within approximately the same number of samples.

4. RSSI varies significantly with the reader-tag distance and tag orientation. These relationships can be accurately modeled by fitting the transmission model of RFID communication with a parameterized empirical model.

By pre-screening the RFID tags to be used and removing tags that report unusually low or high RSSI, measurement errors caused by manufacturing variations can be substantially reduced. RSSI measurement errors can be further decreased by recording the average RSSI from multiple reads. The appropriate number of RSSI measurements to average is determined by the desired accuracy and available sampling time. With the aforementioned steps taken to ensure accurate data collection, the constants for Equation (7) can be calculated, yielding an equation to accurately estimate the expected RSSI for a tag at a given location and orientation.

\section{LOCALIZATION EXPERIMENTS}

With a model for the expected RSSI at a given tag position, it is possible to localize an RFID reader antenna within a field of RFID tags at known locations. In this section, we present an experiment to localize a reader using Equation (7) combined with the maximum likelihood search method described in [8].

\subsection{Localization Algorithm}

The search area to be considered is an $N_{x} \mathrm{x} N_{y}$ rectangular grid of possible locations for the reader. For a set of $N_{T}$ tags, let $\boldsymbol{\Phi}$ be an $N_{T} \times N_{x} N_{y}$ dictionary matrix where the $k$ th column of $\boldsymbol{\Phi}$ is expressed as

$$
\boldsymbol{\phi}_{k}=\left[\phi_{1}^{k}, \phi_{2}^{k} \ldots, \phi_{N_{T}}^{k}\right]^{T}, k=1, \ldots, N_{x} N_{y},
$$

where $(.)^{T}$ denotes vector transpose. The element $\phi_{i}^{k}$ is the expected RSSI measurement at the $k$ th hypothetical reader grid position $\left(x_{k}, y_{k}, z_{k}\right)$ and the $i$ th tag located at $\left(x_{i}, y_{i}, z_{i}\right)$. In this experiment, the reader antenna will be placed at a fixed height $z_{\mathrm{k}}=h$, and all tags will be placed on the same reference plane with height $z_{i}=0$. In this case, the expected RSSI measurement table can be populated with $\phi_{i}^{k}=\operatorname{RSSI}\left(d_{i}^{k}, \theta_{i}^{k}\right)$ given in Equation (6), where

$$
d_{i}^{k}=\sqrt{\left(x_{k}-x_{i}\right)+\left(y_{k}-y_{i}\right)+h^{2}},
$$

and $\theta_{i}^{k}$ is calculated from Equation (3) as the angle between the tag axis and the tag-reader line of sight. With the expected RSSI matrix $\boldsymbol{\Phi}$ calculated, localization of the reader is performed by comparing an input RSSI measurement vector to the columns of $\boldsymbol{\Phi}$. The measurement vector $\mathbf{y}$ contains RSSI data for $N_{T}$ tags averaged over a predetermined number of reads. The elements of $\mathbf{y}$ are arranged to correspond to the rows of $\boldsymbol{\Phi}$. Note that not all of the tags will successfully communicate with the reader during a reading attempt. Tags with no response should be removed from the RSSI measurement vector $\mathbf{y}$, and their corresponding rows of $\boldsymbol{\Phi}$. The $k$ th column of $\boldsymbol{\Phi}$ is then found by solving

$$
\arg \min _{k}\left\|\mathbf{y}-\boldsymbol{\phi}_{k}\right\| .
$$

For large and/or very fine localization grids, computation time can be greatly reduced for real-time tracking applications by limiting $k$ (and subsequently $\left(x_{k}, y_{k}, z_{k}\right)$ ) to positions within a certain radius of the previous localization estimate. 


\subsection{Localization Experiment}

To test the localization algorithm described in [8], we perform experiments to measure localization accuracy with various arrangements and quantities of tags placed around the reader. For each trial, the reader antenna is placed $1.52 \mathrm{~m}$ (60 inches) above the tag plane. The tags are arranged in pairs forming a " + "structure so that they are orthogonal to each other. This method of creating orthogonal pairs or "super-tags" is discussed in [11] as a method of improving localization performance. If one tag of the pair is in an orientation of low directive gain, then the orthogonal tag will be oriented for high directive gain, thus increasing the likelihood of detecting a tag at a given tag site. RSSI results respect to different tag orientations also provides important information for reader localization. For each tag arrangement, 100 RSSI measurements per tag are collected to ensure a highly repeatable result. A graphical representation of the experimental setup is given in Fig. 8(a), showing the tag pairs and reader antenna location in the $(x, y)$ plane. The localization algorithm is performed for a given set of RSSI measurements and the difference between $\mathbf{y}$ and $\boldsymbol{\phi}_{k}$ is calculated at each point on the grid of $0.32 \mathrm{~cm}(1 / 8 \mathrm{inch})$ resolution. For visualization, Fig. 8(b) displays the search space overlaid with a color map, showing the likelihood of the reader being found at each given location in that space. The $k$ th position that gives the smallest $\left\|\mathbf{y}-\boldsymbol{\phi}_{k}\right\|$, shown as a red dot in Fig. 8(b), is chosen as the reader position estimate. In this experiment, the estimation error is $14 \mathrm{~cm}$ (5.5 inches).

We localize the reader in 6 different tag arrangements using 100 RSSI reads to test the accuracy of the position estimates. Fig. 9 shows the results of these experiments. The position estimation error ranges from $7.1 \mathrm{~cm}(2.8$ inches $)$ to $25.4 \mathrm{~cm}$ (10 inches). From these experiments it can be observed that symmetry tag arrangements result in less estimation error, while highly asymmetric arrangements, such as those depicted in Figs. 9(a) and 9(e), result in a higher estimation error.

As discussed in Section 3.2, a reasonable estimate of the true RSSI can be achieved by averaging a small number of RSSI measurements. Repeated localization experiments with 5-, 10-, and 15-point averages are shown in Fig. 10.

\subsection{Discussion of Results}

As shown in Fig. 9, with the proposed localization algorithm we are able to estimate the reader position with a high degree of accuracy. The accuracy appears to be affected most by having strongly asymmetric distributions of tags. Increasing the number of RSSI measurements to average gives an increase in localization accuracy at the expense of sampling time.

\section{CONCLUSION}

In this paper, we have performed experimental studies for accurately localizing an RFID reader in a field of tags. This proposed localization method is based on a model of RSSI as a function of distance and tag orientation, which was derived from measurement data on the behavior of RFID tags. The experimental studies reveal the importance of prescreening tags to ensure uniform tag performance, and averaging a sufficient number of RSSI measurements to overcome measurement inaccuracies. The proposed localization method allows simple and accurate RFID localization.

\section{ACKNOWLEDGMENT}

This work is supported in part by the Office of Naval Research under contract N00014-14-C-0005.

\section{REFERENCES}

[1] R. Want, "An introduction to RFID technology," IEEE Pervasive Computing, vol. 5, no. 1, pp. 25-33, 2006.

[2] D. Hahnel, W. Burgard, D. Fox, K. Fishkin, and M. Philipose, "Mapping and localization with RFID technology," in Proceedings of IEEE International Conference on Robotics and Automation, Barcelona, Spain, pp. 1015-1020, April 2004.

[3] P. V. Nikitin and K. S. Rao, "Theory and measurement of backscattering from RFID tags," IEEE Antennas and Propagation Magazine, vol. 48, no. 6, pp. 212-218, 2006.

[4] D. M. Dobkin, The RF in RFID: UHF RFID in Practice. Newnes, 2012.

[5] Y. Zhang, X. Li, and M. G. Amin, "Principles and techniques of RFID positioning," in M. Bolic, D. Simplot-Ryl, and I. Stojmenovic (eds.), RFID Systems, Research Trends and Challenges, John Wiley, 2010. 


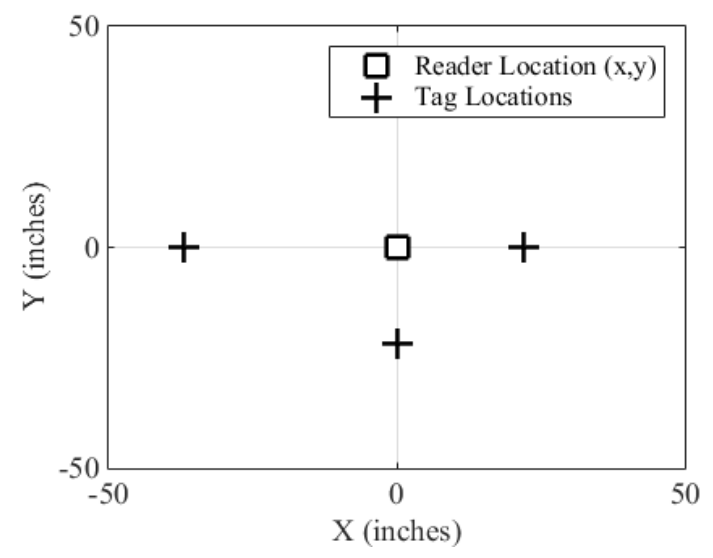

(a)

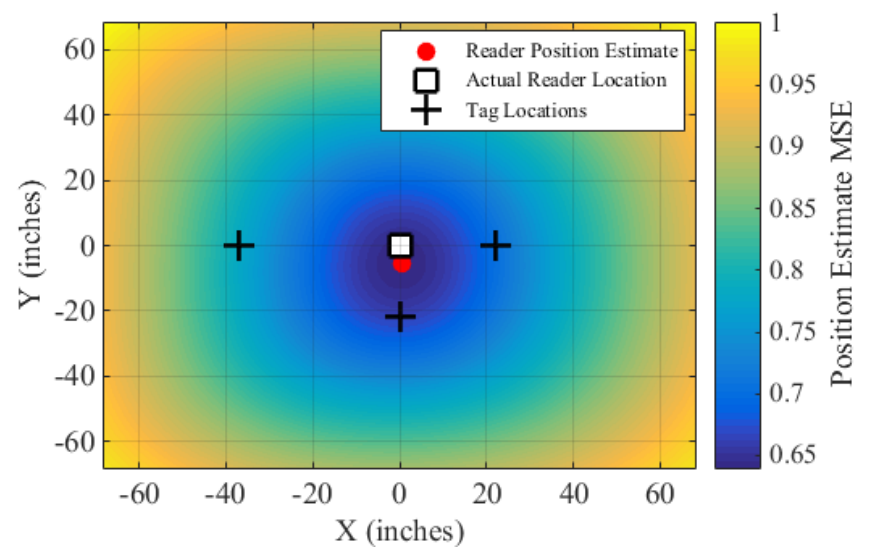

(b)

Figure 8. An example of the reader and tag arrangment for a localization experiment. (a) The reader positioned in a field of three supertags marked by a "+"; (b) The same experiment overlaid with a map of the normalized distance $\left\|\mathbf{y}-\boldsymbol{\phi}_{k}\right\|$. The red dot shows the estimated reader position.

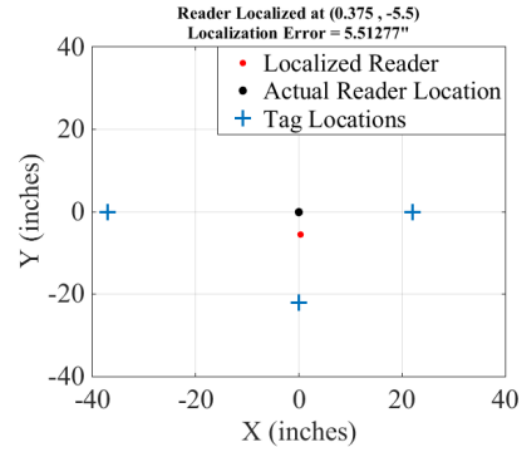

(a)

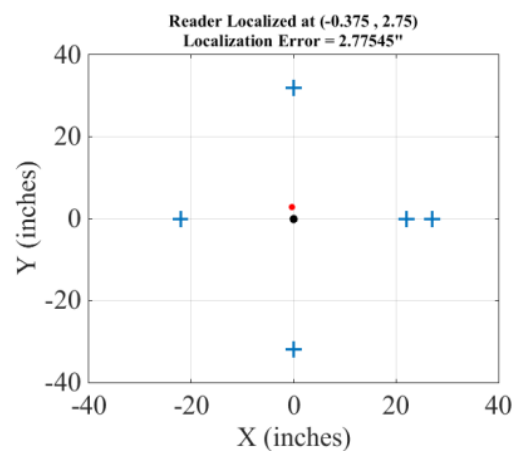

(d)

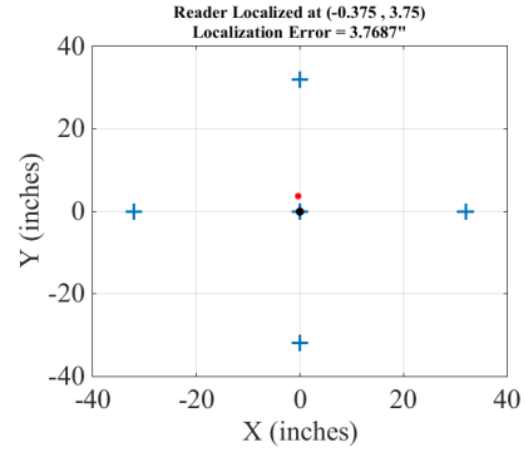

(b)

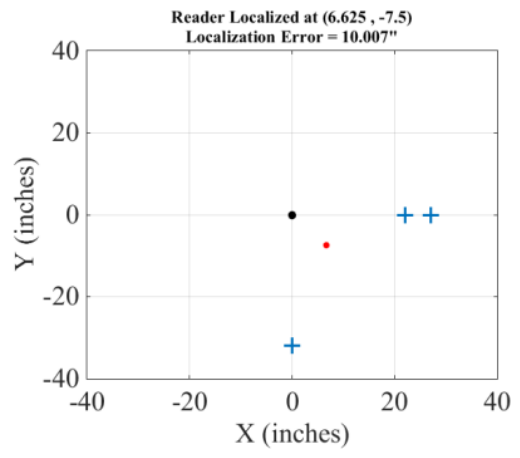

(e)

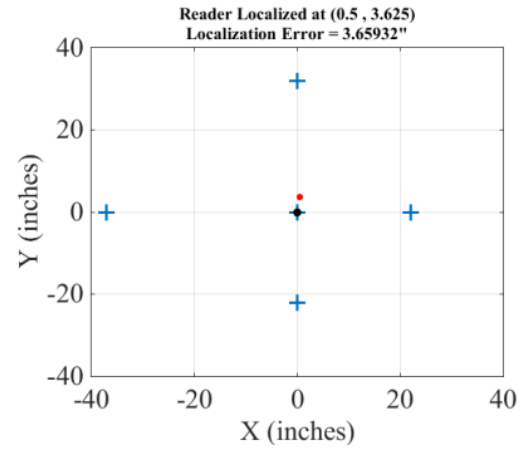

(c)

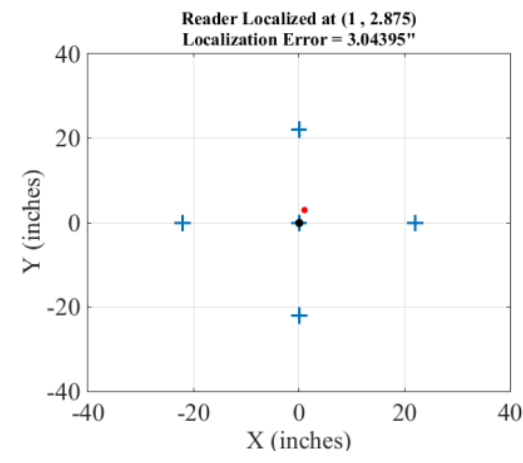

(f)

Figure 9. Results from 6 localization experiments with varying tag arrangements. + symbol denotes a "super tag" pair. 
[6] Y. Zhang, M. G. Amin, and S. Kaushik, "Localization and tracking of passive RFID tags based on direction estimation," International Journal of Antennas and Propagation, vol. 2007, Article ID 17426, 9 pages, Dec. 2007.

[7] L. M. Ni, Y. Liu, Y. C. Lau, and A. P. Patil, "LANDMARC: indoor location sensing using active RFID," in Proceedings of IEEE International Conference on Pervasive Computing and Communications, pp. 407-415, DallasFort Worth, TX, March 2003.

[8] S. Subedi, Y. D. Zhang, and M. G. Amin, "Precise RFID localization in impaired environment through sparse signal recovery," in Proceedings of SPIE Defense, Security, and Sensing, Baltimore, MD, vol. 8753, May 2013.

[9] D. Joho, C. Plagemann, and W. Burgard, "Modeling RFID signal strength and tag detection for localization and mapping," in Proceedings of IEEE International Conference on Robotics and Automation, Kobe, Japan, May2009.

[10] K. Chawla, C. McFarland, G. Robins, and C. Shope, "Real-time RFID localization using RSS," in Proceedings of IEEE International Conference on Localization and GNSS, pp. 1-6, Turin, Italy, June 2013.

[11]P. Asadzadeh, L. Kulik, and E. Tanin, "Gesture recognition using RFID technology," Personal and Ubiquitous Computing, vol. 16, no. 3, pp. 225-234, 2012.

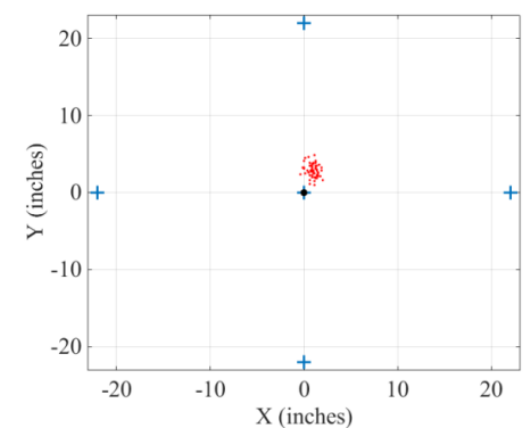

(a)

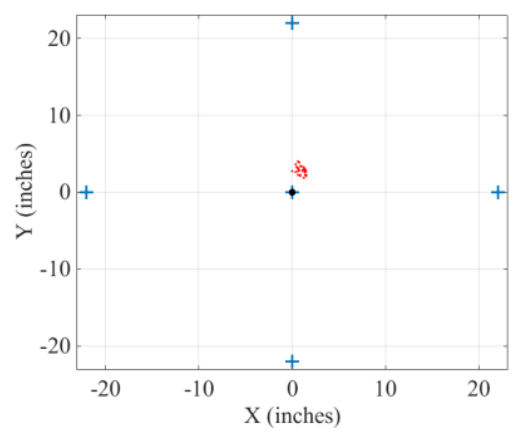

(b)

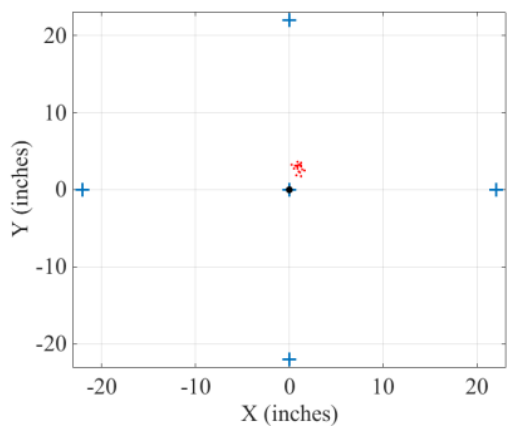

(c)

Figure 10. Reader location estimates with (a) 5, (b) 10, and (c) 15 RSSI measurement averages (red dot: localized reader position; +: super-tag position. The reader is marked with a black dot at $(0,0)$ ).

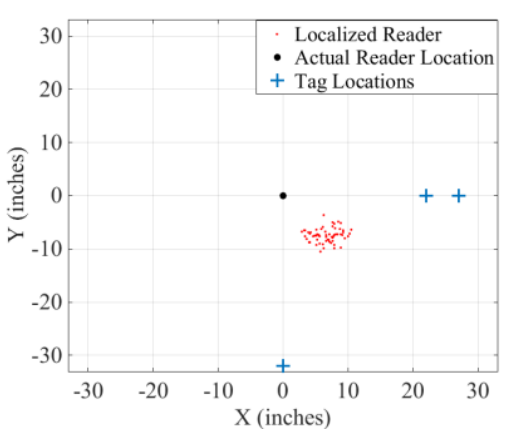

(a)

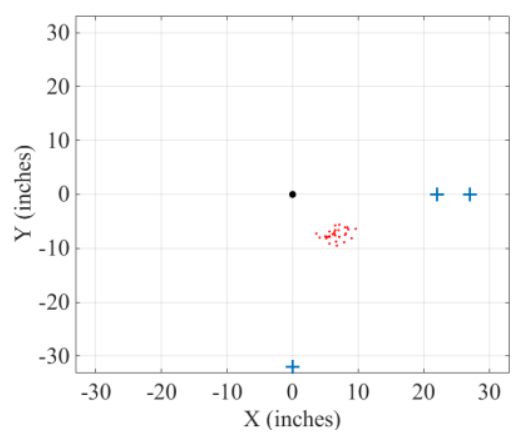

(b)

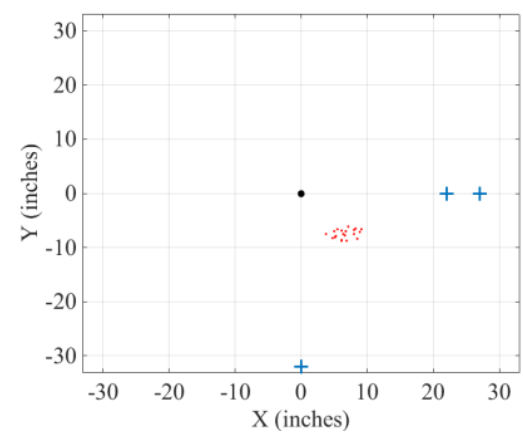

(c)

Figure 11. Reader location estimates with (a) 5, (b) 10, and (c) 15 RSSI measurement averages. 